This is the peer-reviewed version of the article

Filipović, N., Stevanović, M., Nunić, J., Cundrič, S., Filipič, M., Uskoković, D., 2014. Synthesis of poly ( $\varepsilon$-caprolactone) nanospheres in the presence of the protective agent poly(glutamic acid) and their cytotoxicity, genotoxicity and ability to induce oxidative stress in HepG2 cells. Colloids and Surfaces B: Biointerfaces 117, 414-424. http://dx.doi.org/10.1016/j.colsurfb.2014.03.015

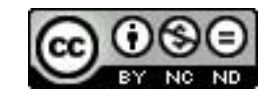

This work is licensed under 


\title{
Synthesis of poly(E-caprolactone) nanospheres in the presence of the protective agent poly(glutamic acid) and their cytotoxicity, genotoxicity and ability to induce oxidative stress in HepG2 cells
}

\author{
Nenad Filipovic ${ }^{a}$, Magdalena Stevanovic ${ }^{a, 1}$, Jana Nunić ${ }^{b}$, Sandra Cundričc , Metka Filipič ${ }^{b}$, \\ Dragan Uskokovic ${ }^{a}$
}

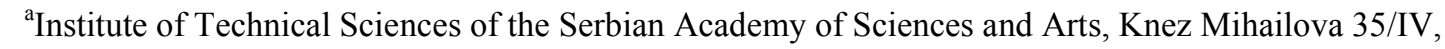
Belgrade 11000, Serbia

${ }^{b}$ Department of Genetic Toxicology and Cancer Biology, National Institute of Biology, Ljubljana, Slovenia ${ }^{\mathrm{c}}$ Biotechnical Faculty, University of Ljubljana, Ljubljana, Slovenia

\begin{abstract}
Nanospheres of poly ( $\varepsilon$-caprolactone) (PCL) with sizes smaller than $200 \mathrm{~nm}$ were produced by combining the freeze drying method and the physicochemical solvent/non-solvent approach. The influence of various types of cryoprotectants (poly(glutamic acid) (PGA) or sacharose) and their concentrations on the outcome of freeze-dried poly(E-caprolactone) particles was evaluated. The physiochemical properties, structural and morphological characteristics of thereby obtained PCL particles were determined by scanning electron microscopy (SEM) and Fourier transform infrared spectroscopy (FTIR). The cytotoxicity of the samples was examined by the 3-(4,5-dimethylthiazol-2-yl)-2,5-diphenyltetrazolium bromide assay (MTT assay). The formation of intracellular reactive oxygen species was measured spectrophotometrically using a fluorescent probe (DCFH-DA assay). In addition, the genotoxic response of PCL particles obtained using PGA as a cryoprotectant was investigated by the Comet assay. This paper focuses on the role of PGA in the synthesis of PCL particles and demonstrates that PGA plays a dual role in the synthesis, i.e. it acts as a stabilizer but also as a cryoprotective agent. The sufficient and optimal concentration of PGA for producing uniform, spherical but also biocompatible PCL nanoparticles is established to be $0.05 \%$.
\end{abstract}

Keywords: poly(glutamic acid), cryoprotectants, poly( $\varepsilon$-caprolactone), nanospheres, biocompatibility

\footnotetext{
${ }^{1}$ Corresponding author: Magdalena Stevanović; tel.: +381-11-2636-994; fax:+381-11-2185-263 Institute of Technical Sciences of the Serbian Academy of Sciences and Arts

Knez Mihailova 35/IV, 11000 Belgrade, Serbia

E-mail address: magdalena.stevanovic@itn.sanu.ac.rs; magdalena.stevanovic@gmail.com
} 


\section{INTRODUCTION}

Aliphatic polyesters have proved to be excellent drug carriers due to their numerous specific properties [1-4]. In this group of polymers, poly( $\varepsilon$-caprolactone) (PCL) has found the most diverse applications. High permeability to small drug molecules, failure to generate an acidic environment during degradation, compared to polylactides and glycolides, and a slow degradation rate are just some of the advantages of PCL [5, 6]. Several different forms of this FDA approved polymer have been previously synthesized and reported to be very efficient drug carriers, such as microspheres, nanocapsules, fibers, films, hydrogels, micelles etc. [5-11]. Micro/nanospheres are the most frequently used forms for therapeutic purposes. Different procedures for preparing these PCL particles have been described and all of them have the same goal - the production of uniform, spherical, stable and non-agglomerated particles [5-11]. In order to achieve this, all parameters must be under strict control during the synthesis. An ideal and universal method does not exist and each procedure has both advantages and disadvantages.

The parameters that usually represent a challenge in designing spherical particles are the nature and concentration of the stabilizers. These, usually organic molecules are used to achieve a desired morphology, prevent the agglomeration of the obtained particles and thereby enhance their stability. Another parameter, which has proved to be of great importance for the stability of the obtained particles, is the drying method. Since nanoparticles are usually prepared in a liquid medium that can offer a favorable environment for the stabilization of their high surface energy, it is important to convert these nanoparticles into a solid state and enhance their long-term stability [12]. However, this step in the synthesis may also affect the particle size and morphology.

In the past few decades, freeze drying has become an irreplaceable drying technique for preparing colloidal polymeric nanoparticles and nano-suspensions, [13]. This method, also known as lyophilization, is a process based on water removal from a frozen sample by sublimation and desorption under vacuum. It has been reported that many components of nanoparticles' formulation have a crucial influence on their resistance to different stress conditions during freeze-drying, and the type and concentration of the cryoprotectant, the nature of the surfactant, the chemical groups attached to the surface of nanoparticles are some of the examples [14].

Several studies have reported that sugars (trehalose, sucrose, glucose, mannitol, etc.) are necessary additives in successful lyophilization of PCL [13, 15-18]. In these studies, the concentrations of sugars used to protect particles from the freezing and drying stress varied from 2.5 to $20 \%(\mathrm{~m} / \mathrm{w})$. However, higher amounts of the used cryoprotectants, usually need to be removed afterwards, which complicate the synthesis procedure. Therefore, it is always desirable to design micro/nano spheres without additives that improve lyophilized products. Finally, efforts to obtain a desired structure are futile if the biocompatibility of the obtained system is compromised in any way. Toxicity is a factor that must be considered before selecting the material used in pharmaceutical formulations. In order to obtain a desired morphology, scientists sometimes use solvents and excipients (surfactants, capping agents, cryoprotectans, etc.) in higher concentrations, which can be toxic. There is a substantial risk of retention of these substances in the final system, leading to its cytotoxicity. In addition, numerous recent studies have underlined concerns about the long-term adverse 
effect associated with the use of nanomaterials in humans, raising the safety issues [19]. It is therefore very important to identfy toxicity and safety risks, as well as risks involved in the use of nanoparticles for in vivo applications.

Poly(glutamic acid) (PGA) is a naturally occurring homopolyamide, with very attractive properties, such as nontoxicity, water solubility, biodegradability, etc., which make it applicable in the broad range of industrial fields (medicine, pharmacy, food, etc.). One of its properties of interest, which is mainly used in food industry, is the antifreeze activity. The cryoprotective effect of amino acids and their salts have been well recognized and reported $[20,21]$. There are many theories that explain why these acids have such cryoprotective properties. Most of them are based on the depression of both the freezing point and the ice fraction of frozen materials by Coulomb's force of the dissociating counterions [22]. Along with being used as polyelectrolytes, these polymers can also be applied as stabilizers of micro/nanoparticles. Furthermore, PGA can enhance bioadhesion and provide additional functional groups to incorporate certain functionalities to nanoparticles, such as the attachment of imaging or targeting agents etc. [23-25]. Apart from being a polyelectrolyte, PGA is also used as capping agent or a stabilizer for a broad range of colloidal particles, i. e. silver nanoparticles [26, 27]. PGA is a highly anionic agent. Anionic agents act by coating the particle surface as the result of an attraction to the positive charges that develop on particles when in contact with water, due to adsorption. Adsorption imparts a net negative charge on the particle surface; accordingly, particles are repelled from each other. In this respect, the described principle can be used to significantly improve the function of PCL particles. Literature data suggest that the incorporation of PGA into an antigenic formulation, influenza vaccine, gene transfection carrier, etc. has led to improved pharmaceutical efficacy. For example, Chan and coworkers have reported that the negatively-charged poly( $\gamma$-glutamic acid)-b-poly(epsiloncaprolactone)-b-poly( $\gamma$-glutamic acid) block copolymer with an encapsulated anti-cancer drug in the presence of healthy tissues leads to a lower cellular uptake. On the other hand, it becomes more hydrophobic in the acidic environment of cancerous tissues, increasing its cellular uptake through the lipid bilayer [28].

Therefore the main objectives of this study were to investigate if PGA can have both stabilizing and cryoprotective effects during the preparation of PCL nanospheres by combined physicochemical solvent/nonsolvent method and freeze drying. Furthermore, we studied the biocompatability of these PCL nanospheres in vitro. The influence of PCL particles, obtained with different concentrations of PGA, on viability of human hepatoma cell lines (HepG2), formation of intracellular reactive oxygen species (ROS), as well as genotoxic responses was investigated.

\section{MATERIALS AND METHODS}

\subsection{Chemicals}

Poly( $\varepsilon$-caprolactone) (ester terminated, 0.55-0.75 dL/g) was purchased from Durect, Lactel (Birmingham, Alabama, USA). Poly(L-glutamic acid) (PGA) with $\mathrm{Mw}=20-40 \mathrm{kDa}$ (99.9\% HPLC purity) was obtained from Guilin Peptide Technology Limited (China) and $\mathrm{D}(+)$ saccharose from VWR BDH Prolabo (Leuven, Belgium). 
The chemicals used in determining cytotoxicity, formation of reactive oxygen species (ROS) and genotoxicity (Eagle Minimal Essential Medium, penicillin/streptomycin, L-glutamine, phosphate buffered saline (PBS), trypsin, fetal bovine serum, non-essential amino acid solution (100x), MTT (3-(4,5dimethylthiazol-2-yl)-2,5-diphenyltetrazolium bromide), tert-butyl hydroperoxide ( $t$-BOOH), benzo(a)pyrene (BaP), ethidium-bromide solution, dimethyl sulfoxide) were obtained from Sigma-Aldrich (St. Louis, USA). Normal melting-point agarose and low melting-point agarose were provided by Gibco BRL (Paisley, Scotland).

All reagents were of the analytic grade and were used as received without further purification.

\subsection{Preparation of PCL micro/nano particles}

PCL particles were synthesized via the previously described procedure, using the solvent/non-solvent system, with a small modification [29]. Commercial granules of PCL (300 mg) were dissolved in an organic solvent chloroform $(5 \mathrm{ml})$, and after approximately one hour, the non-solvent - ethanol $(20 \mathrm{ml})$ was added rapidly. The diffusion of the dissolved particles on the interface between the solvent and non-solvent medium cause an immediate precipitation and the formation of a milky white solution. Then various concentrations of PGA or a sucrose solution were added drop-wise into the suspension. After one-minute homogenization on a magnetic stirrer, the mixtures were poured into Petri dishes and left in a freezer overnight $\left(\mathrm{t}=15 \mathrm{~h}, \mathrm{~T}=-15^{\circ} \mathrm{C}\right)$ to concentrate to their half volume due to solvent evaporation. Almost the entire amount of chloroform and a large amount of ethanol had been thereby removed from the system, enabling uniform distribution of the remaining ethanol in ice during the freezing process that followed. Furthermore, the thickness of the samples layers in Petri dishes was thereby reduced to only few mm. After that, for the next 12 hours, the samples were lyophilized in the freeze dryer (Christ, alpha 1-2 LDplus, Osterode am Harz, Germany) at the temperature of $-57^{\circ} \mathrm{C}$ (temperature of ice condenser) and under the pressure of 0.37 mbar.

\subsection{Examination of interactions between PCL and used excipients}

The qualitative analysis of the samples was performed by Fourier-transform infrared (FTIR) spectroscopy. The FTIR spectra of the samples were recorded in the range of $400-4000 \mathrm{~cm}^{-1}$ using a Carl Zeiss SPECORD

75 Spectrometer at $4 \mathrm{~cm}^{-1}$ resolution. The measurements of the samples were carried out in order to determine possible interactions between PCL and different excipients (PGA or saccharose).

\subsection{Morphology of PCL particles}

The morphology of the obtained nano and microparticles was analyzed by a scanning electron microscope (SEM) (JEOL JSM-639OLV). For this purpose, the samples were coated with gold using the physical vapor deposition (PVD) process. The covering was performed by a Baltec SCD 005 sputter coater, using $30 \mathrm{~mA}$ current from the distance of $50 \mathrm{~mm}$ during $180 \mathrm{~s}$. 


\subsection{Cell culture}

HepG2 cells were obtained from the European Collection of Cell Cultures (ECACC). Cells were grown in Eagle Minimal Essential Medium containing $10 \%$ fetal bovine serum, $1 \%$ non-essential amino acid solution, $2 \mathrm{mM}$ L-glutamine and $100 \mathrm{U} / \mathrm{ml}$ penicillin plus $100 \mu \mathrm{g} / \mathrm{ml}$ streptomycin at $37^{\circ} \mathrm{C}$ in a humidified atmosphere and $5 \% \mathrm{CO}_{2}$.

\subsection{Determining cytotoxicity of samples - MTT assay}

The cytotoxicity of the samples was determined using 3-(4,5-dimethylthiazol-2-yl)-2,5-diphenyltetrazolium bromide (MTT) according to Mossman [30], with minor modifications [31]. This assay measures the conversion of MTT to insoluble formazan by dehydrogenase enzymes of intact mitochondria of living cells. The HepG2 cells were seeded onto 96-well microplates (Nunc, Naperville IL, USA) at a density of 40,000 cells $/ \mathrm{ml}$ and incubated for 20 hours at $37^{\circ} \mathrm{C} / 5 \% \mathrm{CO}_{2}$ to attach. The medium was then replaced by fresh complete medium containing $0,0.0001,0.001,0.01,0.1$ and $1 \%(\mathrm{v} / \mathrm{v})$ of each individual sample (PCL without PGA, PCL with $0.05 \%$ PGA, PCL with $0.5 \%$ PGA and PCL with 1\% PGA), and incubated for 24 h. In each experiment, a negative control (non-treated cells) and vehicle control (1\% emulsion) were included. MTT (final concentration $0.5 \mathrm{mg} / \mathrm{ml}$ ) was then added and incubated for three hours. The medium containing MTT was removed and the formed formazan crystals were dissolved in DMSO. The optical density (OD) was measured at $570 \mathrm{~nm}$ (reference filter $690 \mathrm{~nm}$ ) by a microplate reading spectrofluorimeter (Synergy MX, Biotek, USA). The viability was determined by comparing the OD of the wells containing the treated cells with those of the non-treated cells. Five replicates per concentration point and three independent experiments were performed.

The statistical significance between the treated groups and the controls was determined by two tailed Student's t-test and $P<0.05$ was considered as statistically significant.

\subsection{Determining intracellular reactive oxygen species formation - DCFH-DA assay}

The formation of intracellular reactive oxygen species (ROS) was measured spectrophotometrically using a fluorescent probe, DCFH-DA, as described by Osseni et al. (1999) [32], with minor modifications [33]. DCFH-DA readily diffuses through the cell membrane and is hydrolyzed by intracellular esterases to nonfluorescent $2^{\prime}, 7^{\prime}$-dichlorofluorescin. In the presence of intracellular ROS it is rapidly oxidized to highly fluorescent $2^{\prime}, 7^{\prime}$-dichlorofluorescein. The DCF fluorescence intensity is proportional to the amount of reactive oxygen species formed intracellularly.

HepG2 cells were seeded at a density of 75,000 cells/ml into 96-well, black, tissue culture-treated microtiter plates (Nunc, Naperville IL, USA) in five replicates. The cells were incubated for $20 \mathrm{~h}$ at $37^{\circ} \mathrm{C} / 5 \%$ $\mathrm{CO}_{2}$ to attach; then $20 \mu \mathrm{M}$ DCFH-DA was added for $30 \mathrm{~min}$ and the excess of DCFH-DA was removed; cells were treated with $0,0.0001,0.001,0.01,0.1$ and $1 \% \mathrm{v} / \mathrm{v}$ of each individual sample (PCL without PGA, PCL 
with $0.05 \%$ PGA, PCL with $0.5 \%$ PGA and PCL with $1 \%$ PGA) in PBS. The negative control (non-treated cells), vehicle control (1\% emulsion) and positive control $(0.5 \mathrm{mM} t$-BOOH) were included in each experiment. For the kinetic analyses of the intracellular ROS formation the plates were maintained at $37^{\circ} \mathrm{C}$ and the fluorescence intensity was determined (at the excitation wavelength of $485 \mathrm{~nm}$ and the emission wavelength of $530 \mathrm{~nm}$ ) every $30 \mathrm{~min}$ during the five-hour incubation using a microplate reading spectrofluorimeter (Tecan, Genios, Austria).

The statistical significance between the treated groups and controls was determined by two tailed Student's t-test and by Kruskal-Wallis one-way analysis of variance (ANOVA) with Dunett's posttest; $P<0.05$ was considered as statistically significant. Three independent experiments with five replicates were performed.

\subsection{Determining genotoxicity - classical comet assay}

HepG2 cells were seeded at a density of $\approx 80000$ cells $/ \mathrm{ml}$ into 12 -well microtiter plates (Corning Costar Corporation, Corning, New York, USA). After incubating the cells at $37^{\circ} \mathrm{C}$ in $5 \% \mathrm{CO}_{2}$ for $20 \mathrm{~h}$ in order to make them attach to the plates, the growth medium was replaced with fresh medium containing $0,0.0001$, $0.001,0.01,0.1$ and $1 \%(\mathrm{v} / \mathrm{v})$ of the samples (PCL without PGA, PCL with $0.05 \%$ PGA, PCL with $0.5 \%$ PGA and PCL with 1\% PGA) and incubated for $24 \mathrm{~h}$. Each experiment included the negative control, the positive control (50 $\mu \mathrm{M} \mathrm{BaP})$ and the vehicle control (1\% emulsion). At the end of the exposure period, cells were harvested and the DNA damage was determined using the protocol proposed by Singh et al. with minor modifications [34]. Images of 50 randomly selected nuclei per experimental point were analyzed using the image analysis software Comet Assay IV (Perceptive Instruments, UK). Three independent experiments were performed for each of the treatment conditions. The percentages of tail DNA were used to measure the levels of DNA damage. One-way analysis of variance (ANOVA, Kruskal-Wallis) was used to analyze the differences between the treatments within each experiment. Dunnet's tests were used to compare median values of percentage tail DNA; $p<0.05$ was considered as statistically significant.

\section{RESULTS AND DISCUSSION}

\subsection{Fourier-transform infrared spectroscopy (FTIR)}

The qualitative characterization of the formed nanoparticles was carried out by FTIR spectroscopy. The details of the spectra interpretation and band assignments for most vibrations are given in Table 1. In general, the samples obtained by adding PGA or saccharose have a noticeable increase of band intensity and shifting of certain bands is observed, which is probably due to the overlapping of the same functional groups with PCL and due to the effect of conjugation caused by bonding of these molecules to the polymer chain. These effects are more pronounced in the samples containing the highest amount of PGA. Therefore, the only visible 
differences in the spectrum of PCL prepared with the addition of $0.05 \%$ PGA were the asymmetric and symmetric $\mathrm{CH}_{2}$ stretching vibrations and the symmetric $\mathrm{COC}$ vibration. There were no changes in the spectrum appearance even when the concentration of PGA was 10 times higher (for this reason data for this sample are not given in Table 1). However, in the sample with the highest concentration of PGA clear evidence for the interaction between the two macromolecules and the adsorption of PGA on the PCL surface could be observed in the splitting of the carbonyl band into two bands at 1710 and $1730 \mathrm{~cm}^{-1}$ (the one at the higher wave number originates from PGA) [35] and the appearance of the new intensive band at $1070 \mathrm{~cm}^{-1}$, which can be attributed to the stretching C-N band of PGA [26]. For the PCL-saccharose sample most of the characteristic bands were blue-shifted. Also, the broad peak at around $3400 \mathrm{~cm}^{-1}$, observed in all previous samples (represents the overtone of the carbonyl stretching band), has outgrown in an intense shoulder. The adsorption of IR radiation on this wave number is characteristic for the $\mathrm{OH}^{-}$stretching vibration and is expected for saccharose.

Table 1. FTIR band position and the assignment for the spectra obtained from four different PCL samples

\begin{tabular}{|c|c|c|c|c|}
\hline \multirow[t]{2}{*}{ Assignment } & \multicolumn{4}{|c|}{$\frac{\text { Band position }}{\underline{\left(\mathrm{cm}^{-1}\right)}}$} \\
\hline & PCL & $\begin{array}{l}\text { PCL+0.05\% } \\
\text { PGA }\end{array}$ & PCL+1\% PGA & $\begin{array}{l}\text { PCL+1\% } \\
\text { Saccharose }\end{array}$ \\
\hline overtones of $C=\mathrm{O}^{[26]}$ & $\sim 3400$ & $\sim 3400$ & $\sim 3400$ & $\sim 3400^{\mathrm{a}}$ \\
\hline symmetric $\mathrm{CH}_{2}{ }^{[36]}$ & 2855 & 2850 & 2850 & 2850 \\
\hline asymmetric $\mathrm{CH}_{2}{ }^{[36]}$ & 2910,2925 & 2920 & 2920 & 2925 \\
\hline stretching $C=\mathbf{O}^{[35,36]}$ & 1710 & 1710 & 1710,1730 & 1710 \\
\hline $\mathrm{CH}_{2}$ vibrations $^{[37]}$ & $\begin{array}{l}1460,1410 \\
1360\end{array}$ & $\begin{array}{l}1470,1410 \\
1360\end{array}$ & $\begin{array}{l}1465,1400 \\
1360\end{array}$ & $\begin{array}{l}1450,1400 \\
1350\end{array}$ \\
\hline $\begin{array}{l}\text { stretching C-O \& C- } \\
C \text { in crystal. phase }{ }^{[36]}\end{array}$ & 1290 & 1295 & 1290 & 1280 \\
\hline $\operatorname{asymmetric}_{\mathrm{COC}^{[36]}}$ & 1235 & 1240 & 1220 & 1210 \\
\hline symmetric $\mathrm{COC}^{[36]}$ & 1160 & 1160 & 1150,1160 & 1150 \\
\hline OC vibrations ${ }^{[37]}$ & $\begin{array}{l}1100,1040, \\
955\end{array}$ & $\begin{array}{l}1105,1040 \\
960\end{array}$ & $\begin{array}{l}1100,1030 \\
950\end{array}$ & $\begin{array}{l}1090,1010, \\
950\end{array}$ \\
\hline stretching $C-N^{[26]}$ & - & - & 1070 & - \\
\hline $\begin{array}{l}\mathrm{CH}_{2} \text { rocking } \\
\text { vibration }\end{array}$ & 720 & 730 & 720 & 710 \\
\hline
\end{tabular}

a - in this sample, the broad and intense shoulder comes from the overtones of carbonyl bands and the stretching vibrations of hydroxyl group. 


\subsection{Morphological characteristics of PCL micro and nanoparticles obtained without and with different protective agents (SEM)}

In order to observe the particle morphology arising from interactions between PCL and PGA or saccharose and drying conditions, SEM was used. Figures 1 and 2 show that there is a significant difference in morphology between the samples prepared without and with different protective agents. PCL particles obtained without cryoprotectants were irregularly shaped, creating large agglomerates (Fig. 1). The right image with higher magnification reveals that flakes were formed on the surface of these agglomerates, which could be attributed to an intense sublimation of ice crystals under high vacuum. It is obvious that the use of excipients is necessary in order to produce spherical particles.
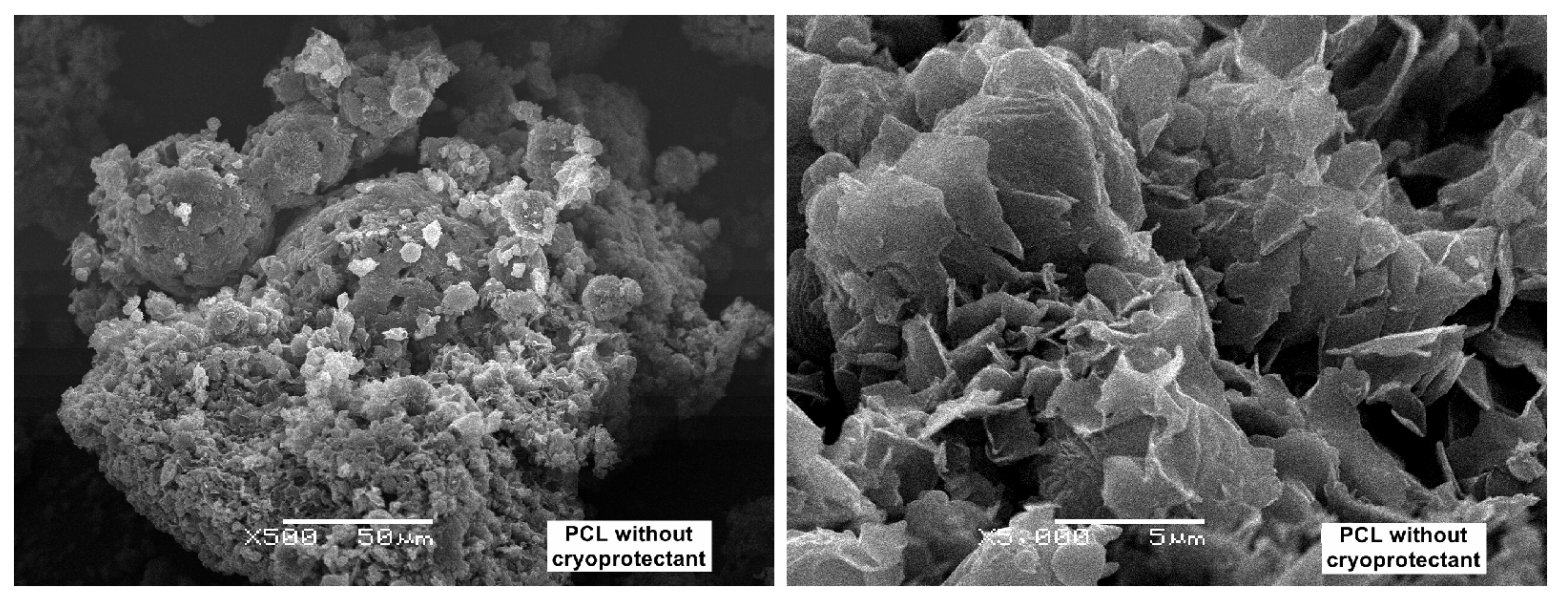

Figure 1. Morphology of PCL particles prepared without excipients.

As we have already mentioned, saccharose is well known for cryoprotective properties, while PGA has an antifreeze activity. According to Arai et al., almost all of the highly antifreeze active substances also have cryoprotective effects [38]. A known problem addressed in the literature, which can occur with the protective agents/additives is its difficult removal from the system [39].Even if the protective agent is biocompatible, its presence may hinder biochemical reactions and reduce drug efficacy [39]. We have previously demonstrated that PGA in the concentration of $0.05 \%(\mathrm{v} / \mathrm{v})$ is an efficient stabilizer of PCL particles [29]. However, lower temperatures reduce the rate of chemical reactions [40]. On the other hand, chemical reactions may actually accelerate in a partially frozen aqueous solution due to an increased solute concentration [41]. For example, the study by Liu and Orgel [42] shows that the oligomerization rate of $\beta$ glutamic acid in the presence of a water-soluble carbodiimide is much quicker at $-20^{\circ} \mathrm{C}$ than it is at 0 or $25^{\circ} \mathrm{C}$ due to solute concentration. Therefore, in order to examine the cryoprotective property of PGA and compare it with widely used saccharose, two samples of PCL were initially prepared, using $1 \%$ solution of either PGA or saccharose. The resulting morphologies are given in Figure 2. 

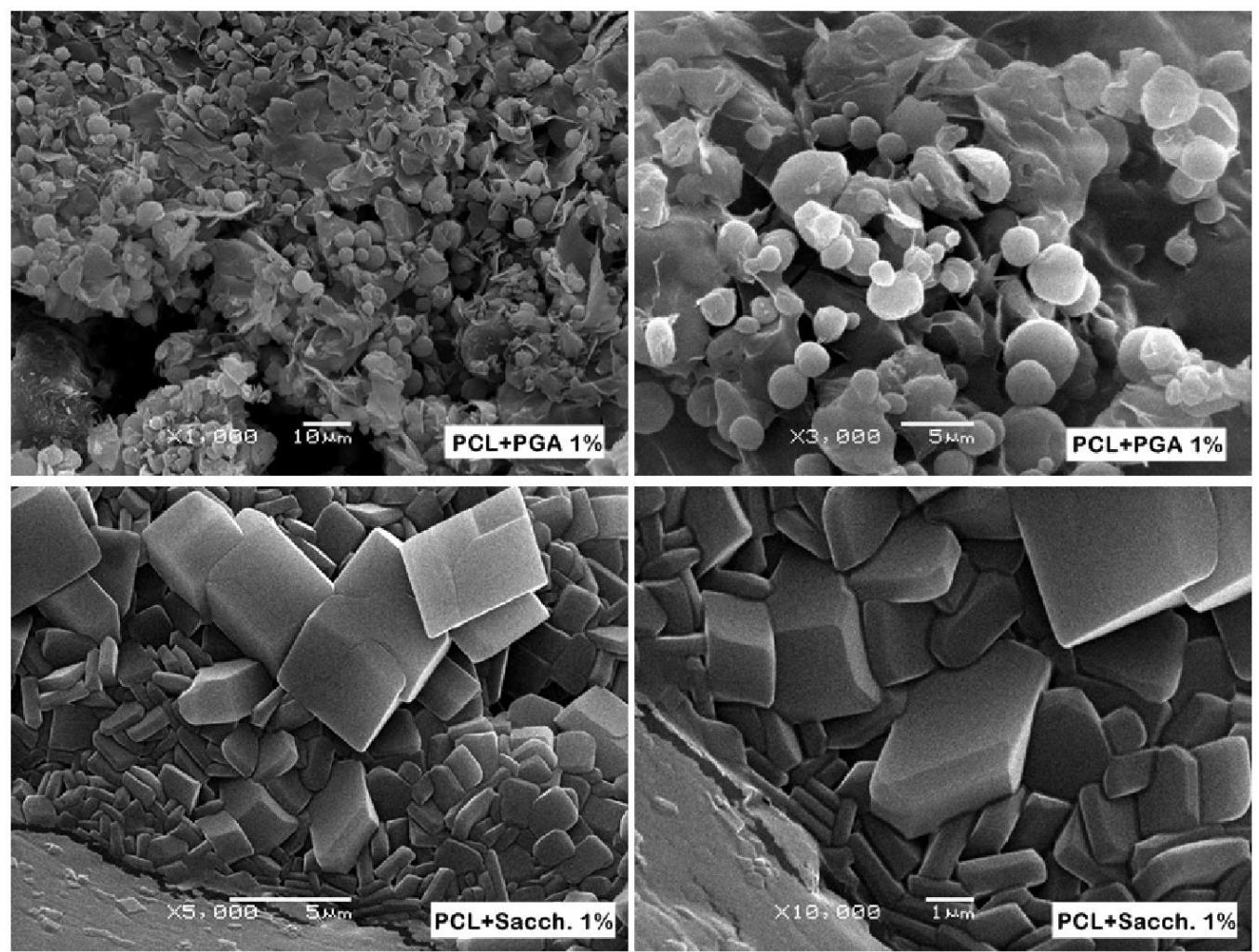

Figure 2. Representative SEM images of PCL particles obtained by adding $1 \%$ solution of PGA (upper row) or $1 \%$ solution of saccharose (lower row).

The huge difference between the samples' morphologies is immediately noticeable. The addition of PGA partly led to the formation of spherical particles, but substantial portion of them were coalesced in irregularly shaped films. Conversely, the sample prepared using the solution of saccharose has a dramatically changed morphology, without any similarity to the previous samples. Polymeric particles formed well-defined cubes and plates of different sizes. Since such particle forms have not been reported in literature either for PCL or saccharose, we believe that during the initial stage of the particle formation, saccharose failed to stabilize; accordingly, this unusual morphology is a consequence of freeze drying conditions, i.e. freezing. The freezing process, which happens very quickly after placing the samples onto the shelves of a freeze dryer, was induced by vacuum [43]. Low pressure favors the evaporation of the solvent and the associated enthalpy of evaporation reduces the local temperature on the solvent surface. This leads to the formation of a thin film of ice. Since the thickness of the samples was previously reduced, the temperature gradient is insignificant and the formation of small ice crystals is favorable. However, the freezing rate could slightly vary according to the different nature of the excipients (Figure 3). At the beginning, polymeric particles are well-dispersed in the suspension. By lowering pressure, the surrounding water gets frozen, which reduces the mobility of each particle of the dispersed phase. The growth of ice crystals follows the direction of the temperature gradient; accordingly, in this case they are expected to be evenly distributed over the entire volume of the samples. At the same time, the molecules of cryoprotectants and polymeric particles become rejected from the ice phase into the small pockets of the cryo-concentrated liquid phase. The homogeneous distribution of the polymer and cryoprotectants in the cryo-concentrated phase is of crucial importance and it depends on many factors: the differences in diffusivity, surface energy, interactions between particles, viscosity etc. [44]. Since 
molecules of saccharose failed to adsorb on the surface of PCL and bearing in mind the large difference in the size of this molecules, the mobility of solutes is significantly different. Also, the rapid freezing and expansion of the formed ice creates mechanical pressure and promotes compaction of polymer particles [45] and thereby additionally increases this difference. This could eventually lead to an inhomogeneous distribution of PCL particles and molecules of saccharose. When the concentration of saccharose is higher than $\sim 75 \%(\mathrm{w} / \mathrm{w})$, further temperature decrease could lead to the formation of a glassy state [46]. The drawback of vitrification is that some amount of water is trapped inside the solid phase and it can be removed only after secondary drying. This small amount of water can provide sufficient adhesion forces that, along with mechanical pressure created due to the expansions of ice crystals, could compress the PCL particles into the shapes of cube and/or plate. Contrary to saccharose and based on the results of FTIR spectroscopy, PGA was able to attach onto the surface of PCL and enhance the possibility for achieving a high degree of homogeneity in ice crystal growth and cryo-concentrated solution. At the same time, the sizes of these molecules are comparable, just like their viscosity and diffusivity. Finally, when the cryo-concentrated liquid phase becomes saturated, it will solidify and immobilize the PCL particles keeping their size and shape fixed. It is also possible that the cryo-concentrated phase solidify through crystallization, which promotes the freezing of all water and therefore it facilitated removing.

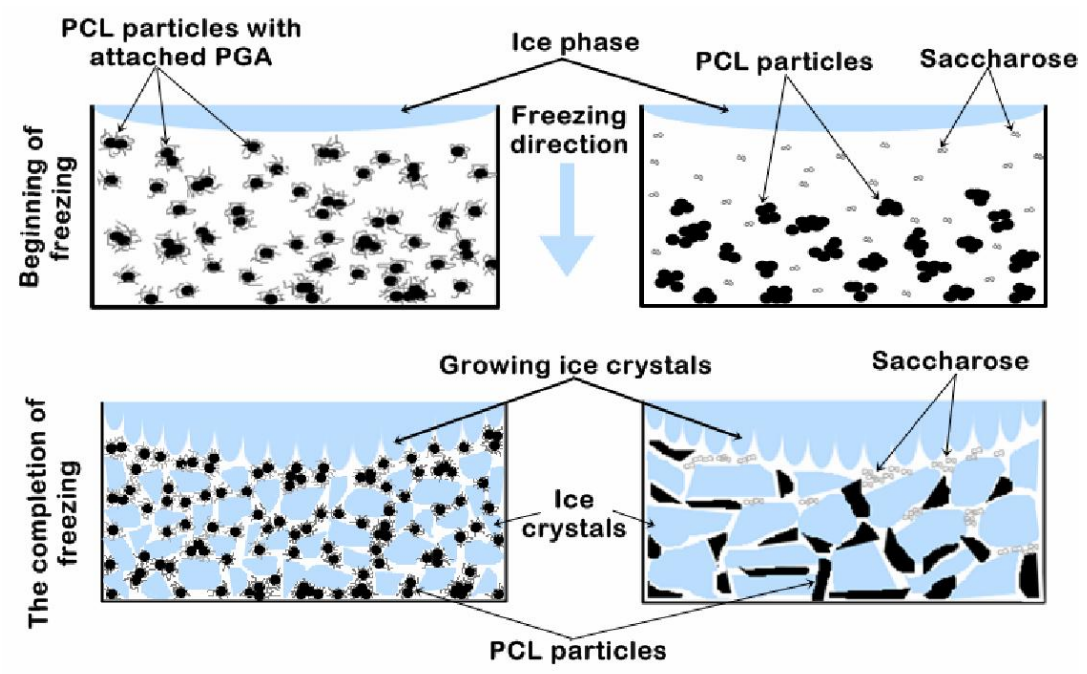

Figure 3. Scheme representing the proposed particles behavior during the freezing process for PCL+PGA and PCL+saccharose samples.

After the comparison of the samples prepared using different cryoprotectants, further investigations were carried out only with PGA, since our goal was to produce uniform spherical particles. Two new samples were prepared by decreasing the concentration of PGA to $0.5 \%$ and $0.05 \%$. The morphologies of the obtained particles are given in Fig. 4. It is evident that improvement of morphology is inversely dependent on the PGA concentration. Thus, the best morphology is observed in the system prepared with the $0.05 \%$ solution of PGA. These particles were spherical, with a smooth surface and sizes below $200 \mathrm{~nm}$ (Figure 4C). The number-based mean particle size for this sample obtained from SEM images (350 counted particles) is $168 \pm 16 \mathrm{~nm}$. The 
image analysis was carried out using the Particle Size Analyzer macro (R. Sperling, ICN, Spain) for the ImageJ software (W. Rasband, NIH, US).

In the sample containing a 10 times higher concentration of PGA, generally spherical morphology is noticed, but particles are larger and have diverse dimensions. Also, in certain parts of the sample, the formation of thin continuous layers of the polymer is observed. It seems that at this concentration, the maximum amount of the stabilizer which ensures the stability of the system was already exceeded.
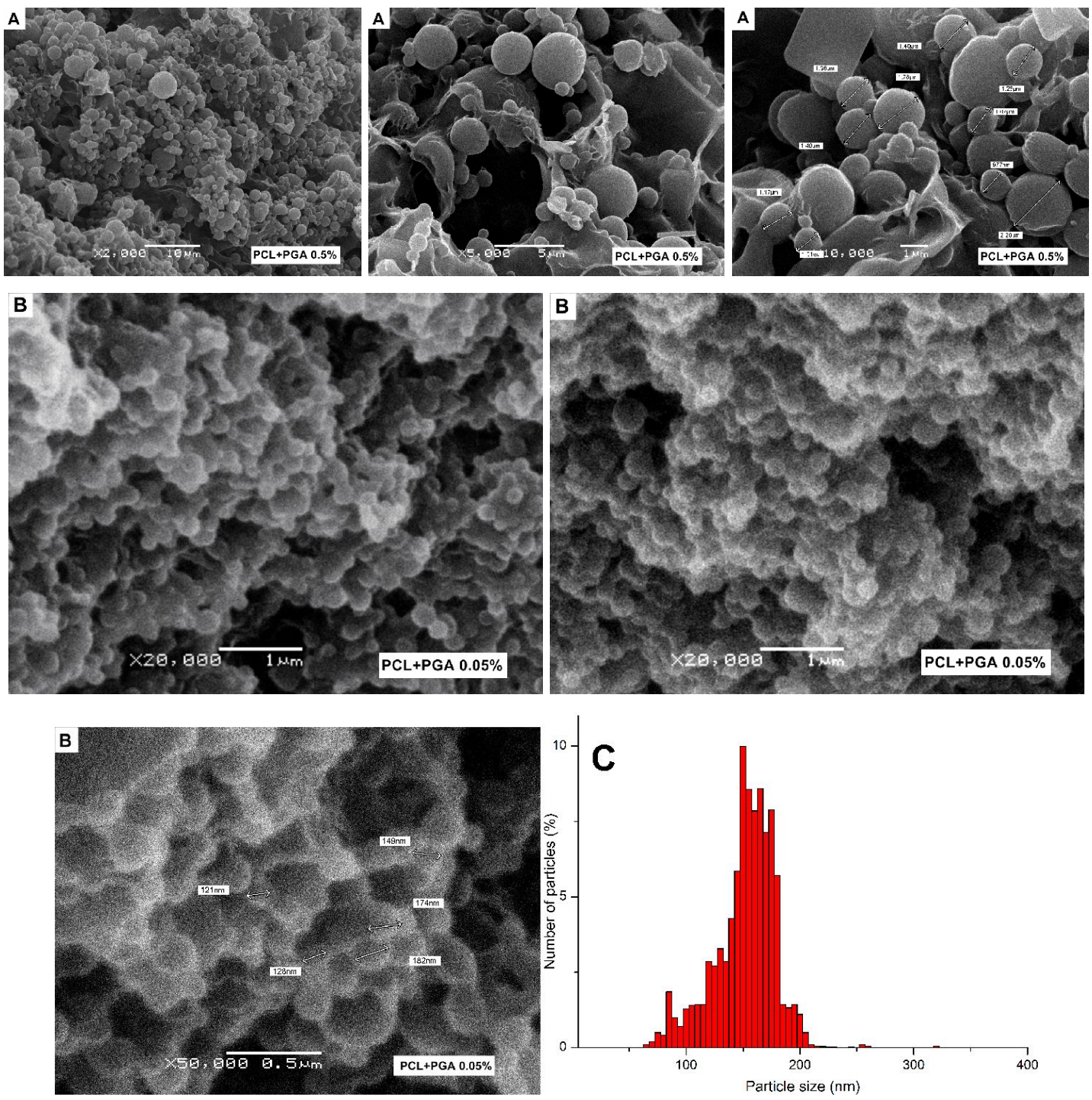

Figure 4. Representative SEM images of PCL particles obtained using PGA as a cryoprotectant where the concentration of PGA was decreased to $0.5 \%$ (A, upper row, bars $10 \mu \mathrm{m}, 5 \mu \mathrm{m}$ and $1 \mu \mathrm{m}$ ) or to $0.05 \%$ (B, lower row, bars $1 \mu \mathrm{m}$ and $0.5 \mu \mathrm{m}$ ) and the size distribution of PCL particles obtained with 0.05\%PGA (C)

Being a water-soluble anionic polyelectrolyte, PGA's stabilizing properties are caused by its electrostatic in nature. This type of stabilization is characteristic for aqueous systems, so its influence decreases with less polar solvents [47]. Thus, for our system there is additional way by which PGA affect the morphology of the PCL particles and it refers to its high molecular weight. For polymers with $\mathrm{Mw}>10000 \mathrm{D}$, the chain dimensions are comparable to the range of the Van der Waals attraction [47]. Generally, two different mechanisms are accepted for polymeric stabilization: steric stabilization and depletion stabilization. 
The first one is due to the adsorption on the dispersed particle, and the second occurs when the particles are free in solution and surrounds dispersed one. Based on the data obtained by FTIR spectroscopy, we suggest that the stabilization with PGA is a result of the combination of the electrostatic and steric mechanisms, i.e. the electrosteric mechanism. Nevertheless, if the particles were left in suspension for a certain time, or dried at room temperature, their coalescence into larger spheres would probably occur. In order to confirm this hypothesis and to determine the influence of the freeze drying conditions on the morphology of the obtained particles, a new sample was prepared but instead of freeze drying, the sample was dried at room temperature and atmospheric pressure for 48 hours. The SEM images of this sample are shown in Fig. 5. Assuming that all other parameters of the particle preparation were strictly controlled, it is evident that the drying temperature and pressure had substantial effect on the particle morphology. Particles were spherical, larger, nonuniform and the presence of agglomerates was observed. Apparently, the evaporation rate and therefore the duration of drying had influence on the size of the obtained particles. In this case, PGA effectively stabilized particles in the initial phase of their preparation, but not during the solvent evaporation. Thus, as the freeze drying theory predicts, with the right choice of protective agent and freezing conditions this method provides improvement in particles size and shape, as well as the improvement of their stability.

Based on the overall data it is difficult to firmly prove the cryoprotective mechanism of PGA. Some of the possible causes could be (i) an increase in viscosity, (ii) increase in super-cooling rate (which ultimately affects the product temperature and the drying time), (iii) the method of solidification or (iv) the combination of all of them [44].
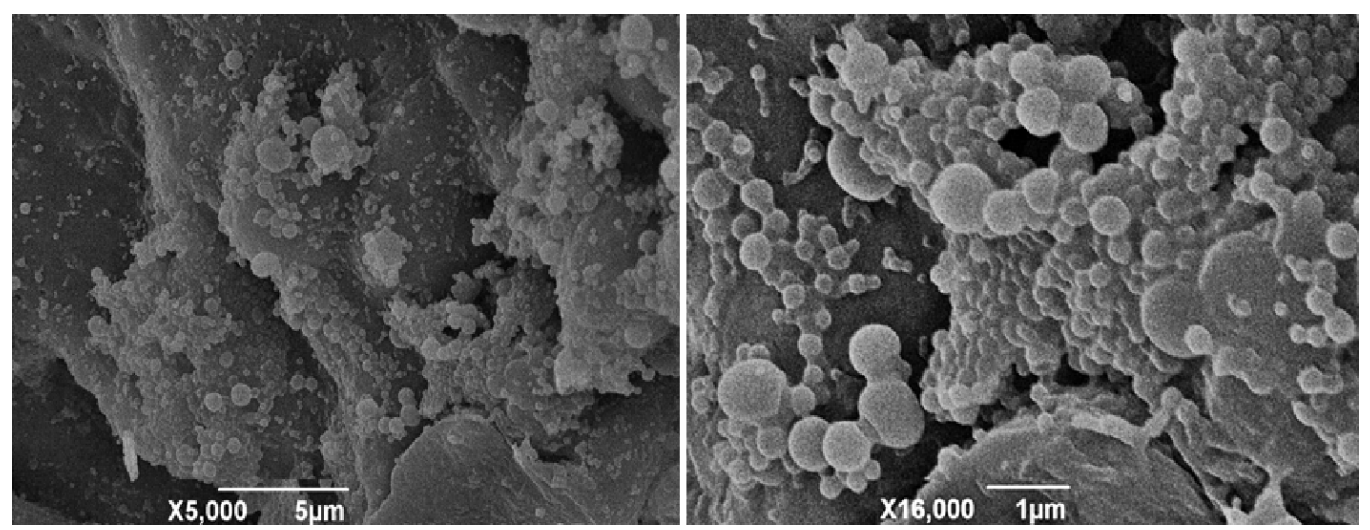

Figure 5. Representative SEM images of PCL particles prepared with $0.05 \%$ solution of PGA and dried at room temperature.

However, the disruption of the stabilizing effect of PGA at increased concentrations was unexpected. A possible explanation could lie in the phenomenon called bridging flocculation [47]. Bridging flocculation takes place when polymer chains are very long and the surface coverage by adsorbed polymer is high. When two polymer-coated surfaces (particles) approach one another, there is an overlap between the adsorbed layers. This in turn causes the polymer concentration to increase locally in the region between particles. If the side chains of the adsorbed polymer are sufficiently close and the right-oriented affinity between them is large enough to overcome the repulsion caused by electrosteric stabilization, then bridging flocculation occurs. At lower concentrations, the possibility for polymer to form adequate bridging links between particles is small. 


\subsection{In vitro cytotoxicity of the samples}

The polymers employed in the preparation of pharmaceutically relevant nanoparticles are generally regarded as safe [48]. However, despite the fact that nanoparticles behave differently from bulk materials, toxicity studies reporting on polymeric nanoparticles are very rare. Therefore, in order to estimate the risk of PCL particles to cause undesirable effects in humans, we first investigated their potential cytotoxicity in an in vitro human cell culture model. Since the main route of potential exposure for PCL particles is oral, and it has been demonstrated that passive targeting to liver and spleen represents the major mechanism for systemic clearance [49], for our study we have chosen liver cells, human hepatoma cell line - HepG2. Over the last decade, many studies have reported that HepG2 cells are a useful in vitro experimental model for the detection and identification of (geno)toxicants [50]. The main advantage of these cells is their retained metabolic activity (activities of various CYPs and some Phase 2 enzymes, involved in metabolism of xenobiotics), due to which they are considered to better reflect the processes in normal human liver than another in vitro toxicity test systems [51].

The cytotoxicity of PCL obtained without cryoprotectant and PCL obtained with different concentrations of PGA as a cryoprotectants was determined with MTT assay. HepG2 cells were exposed to 0, $0.0001,0.001,0.01,0.1$ and $1 \%(\mathrm{v} / \mathrm{v})$ of the samples (PCL without PGA, PCL with $0.05 \%$ PGA, PCL with $0.5 \%$ PGA and PCL with $1 \%$ PGA) for $24 \mathrm{~h}$ (Figure 6).
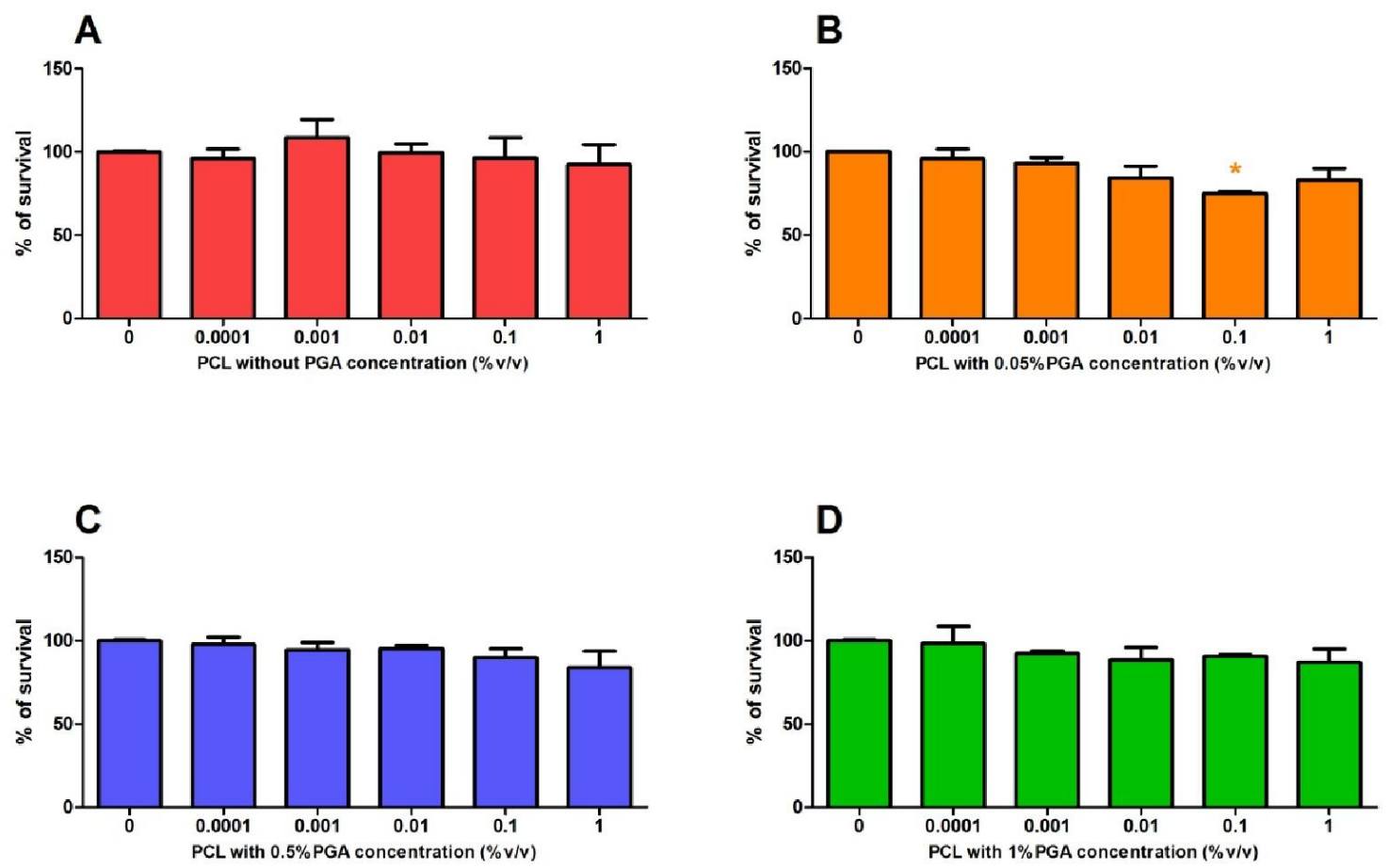

Figure 6. Viability of HepG2 cells treated with increasing doses of PCL without PGA (A), PCL with $0.05 \%$ PGA (B), PCL with $0.5 \%$ PGA (C) and PCL with $1 \%$ PGA (D) for $24 \mathrm{~h}$. The data are presented as mean values of three independent experiments (each with five replicates) \pm S.D. (*) denotes a 


\section{significant difference between the sample-treated groups and vehicle-treated control groups (Student's t-test, $\mathbf{P}<0.05)$.}

The samples of PCL without PGA, PCL with $0.5 \%$ PGA and PCL with $1 \%$ PGA did not significantly reduce the viability of HepG2 cells (Figure 6A, 6C and 6D). The sample of PCL with $0.05 \%$ PGA caused a slight decrease in cell viability ( $\sim 84 \%$ ) only in the cells exposed to $0.1 \%$ of the sample (Figure 6B). Our results are in agreement with study of Campos et al. [52], in which they also with MTT assay did not observe significant cytotoxicity of PCL nanoparticles (an average diameter of these particles has been $450 \mathrm{~nm}$ ) in a test system with Balb-c mouse $3 \mathrm{~T} 3$ fibroblast cells after $24 \mathrm{~h}$ of exposure (concentration not provided). Using the with MTT assay, Singh and Ramarao [49] have demonstrated a slight reduction in cell viability in HepG2 cells at two highest concentrations, 300 and $1000 \mu \mathrm{g} / \mathrm{ml}$, after an exposure to PCL nanoparticles (average particle diameter was $363 \mathrm{~nm}$ ) for $72 \mathrm{~h}$, which they attribute to the intracellular degradation of these nanoparticles. However, PCL degrades much more slowly than other known biodegradable polymers and the samples used in our study were confirmed to be stable; accordingly, the role of degradation in potential toxicity is out of the scope.

\subsection{Induction of intracellular reactive oxygen species by the samples}

Among various stress responses elicited by nanoparticles, the production of reactive oxygen species (ROS) appears to be the earliest event, which is also generally proposed to account for their toxicity [49]. In order to establish the influence of our samples (PCL without PGA, PCL with $0.05 \%$ PGA, PCL with $0.5 \%$ PGA and PCL with 1\% PGA) on intracellular ROS formation, we measured the kinetics of their formation in HepG2 cells using a DCFH-DA assay. HepG2 cells were exposed to increasing doses of the samples (PCL without PGA, PCL with $0.05 \%$ PGA, PCL with $0.5 \%$ PGA and PCL with $1 \%$ PGA) and DCF fluorescence intensity was measured in $30-\mathrm{min}$ intervals during the five hours of incubation at $37^{\circ} \mathrm{C}$. 
A
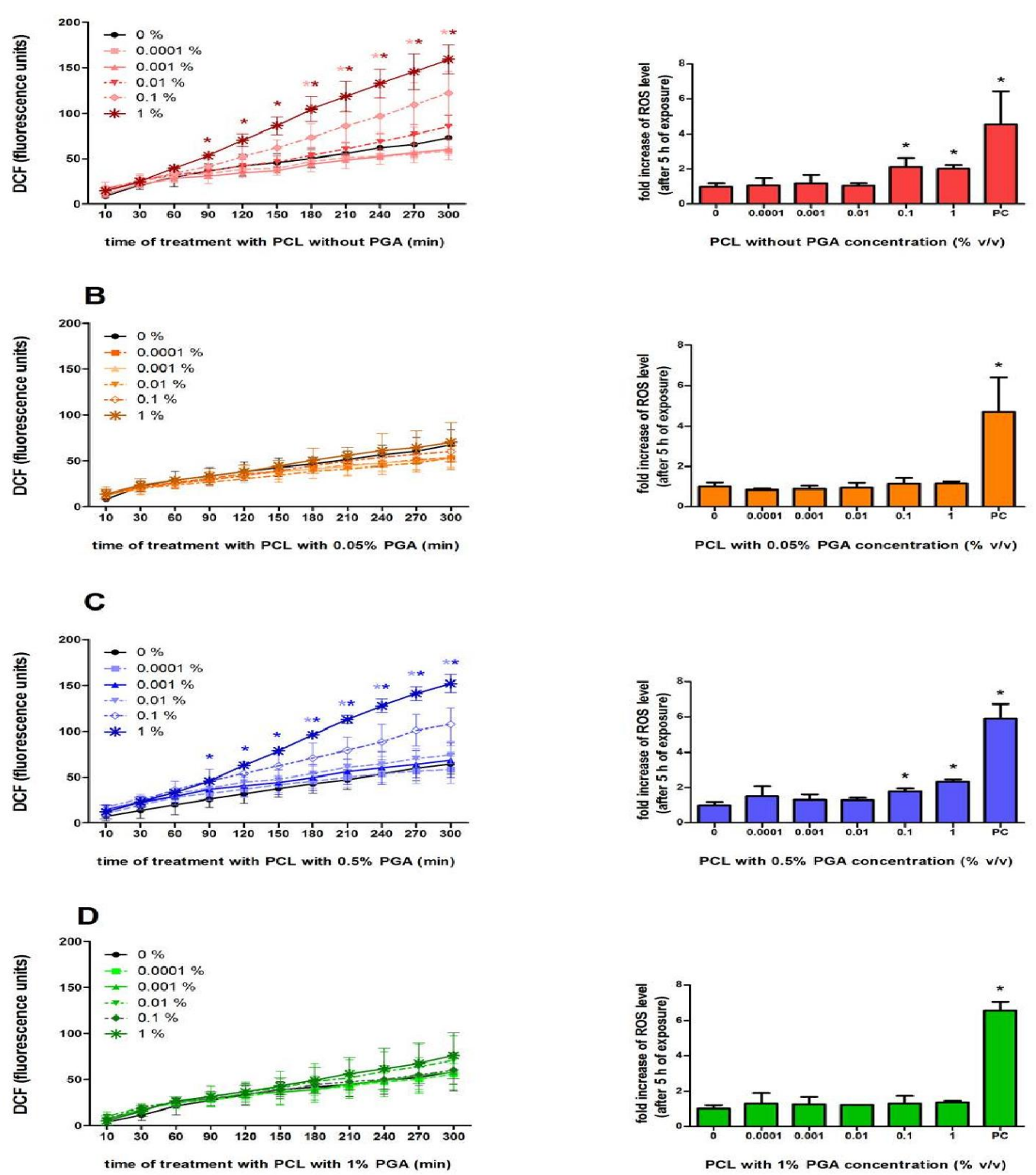

Figure 7. Intracellular ROS formation in HepG2 cells induced by samples (PCL without PGA, PCL with $0.05 \%$ PGA, PCL with $0.5 \%$ PGA and PCL with 1\% PGA). The HepG2 cells were loaded with DCFH-DA $(20 \mu \mathrm{M}$ for $30 \mathrm{~min})$, washed and then exposed to increasing doses $(0,0.001,0.01,0.1$ and 1 $\%$ ) of PCL without PGA (A), PCL with $0.05 \%$ PGA (B), PCL with $0.5 \%$ PGA (C) and PCL with $1 \%$ PGA (D). Exposure to $0.5 \mathrm{mM}$ t-BOOH was used as the positive control (PC). DCF fluorescence intensity was measured at $30 \mathrm{~min}$ intervals during the $5 \mathrm{~h}$ incubation. Left graphs: kinetic of ROS formation during 5 hour exposure; each point represents the mean of five replicates $( \pm$ SD) of representative experiment; $(*)$ denotes a significant difference between sample-treated groups and control (Student's t-test, $P<0.05$ ); right graphs: fold increase of intracellular ROS level in exposed cells over the level in control cells after 5 hour exposure; each bar represent means $( \pm$ SD) of three independent experiments. $(*)$ denotes a significant difference between sample-treated groups and control (Kruskal-Wallis one-way analysis of variance (ANOVA) with Dunett's posttest). 
In HepG2 cells the samples of PCL without PGA and PCL with 0.5\% PGA induced time-dependent increase in the intracellular ROS formation at concentrations 0.1 and $1 \%(\mathrm{v} / \mathrm{v})$ (Figure $2 \mathrm{~A}$ and $\mathrm{C}$ left). After a five-hour exposure, the DCF fluorescence intensity was more than two-fold higher than that in control cells (Figure 7A and C right). The samples of PCL with $0.05 \%$ PGA and PCL with 1\% PGA did not significantly alter the DCF fluorescence intensity (Figure 7B and D). Although both samples, PCL without PGA and PCL with $0.5 \%$ PGA, induced an increased generation of ROS, it can be observed that the amount of DCF fluorescence units and fold increase in the ROS level are higher after the exposure to PCL without PGA. Based on these results we can hypothesize that PGA has a protective effect against PCL-induced generation of ROS; however further studies are needed to investigate this. Our results for PCL nanoparticles without PGA are in concordance with study of Singh and Ramarao [49]. They determined the generation of ROS in the RAW 264.7 cells after 24-hour incubation using a fluorescent probe, DCFDA. PCL nanoparticles had no effect on ROS production up to a $100 \mu \mathrm{g} / \mathrm{ml}$ concentration, whereas $300 \mu \mathrm{g} / \mathrm{ml}$ showed 1.5 - to two-fold stimulation of ROS production. However, it should be taken into account that the concentration of $300 \mu \mathrm{g} / \mathrm{ml}$ was already cytotoxic,; accordingly, the possibility of false positive results under these test conditions should not be riled out. To the best of our knowledge, no data about the generation of ROS induced by PCL nanoparticles with PGA have been reported in literature. The above results suggest that PCL nanoparticles with PGA are generally tolerated by cells and no significant effect on ROS generation is observed.

\subsection{Induction of DNA strand breaks}

The genotoxicity evaluation is an essential part of toxicity studies. Genotoxic agents in the first place cause DNA damage of somatic cells, leading to aging and increased risk of cancer. On the other hand, changes in DNA in the germ cells, lead to the formation of genetic diseases, and contribute to the genetic burden [53]. In order to evaluate the genotoxic potential of PCL nanoparticles we used the comet assay, which is the most commonly used assay for determining the single-and double stranded DNA breaks. We exposed HepG2 cells to graded doses of the samples (PCL without PGA, PCL with $0.05 \%$ PGA, PCL with 0.5\% PGA and PCL with $1 \%$ PGA) for $24 \mathrm{~h}$, and then we performed a comet assay.
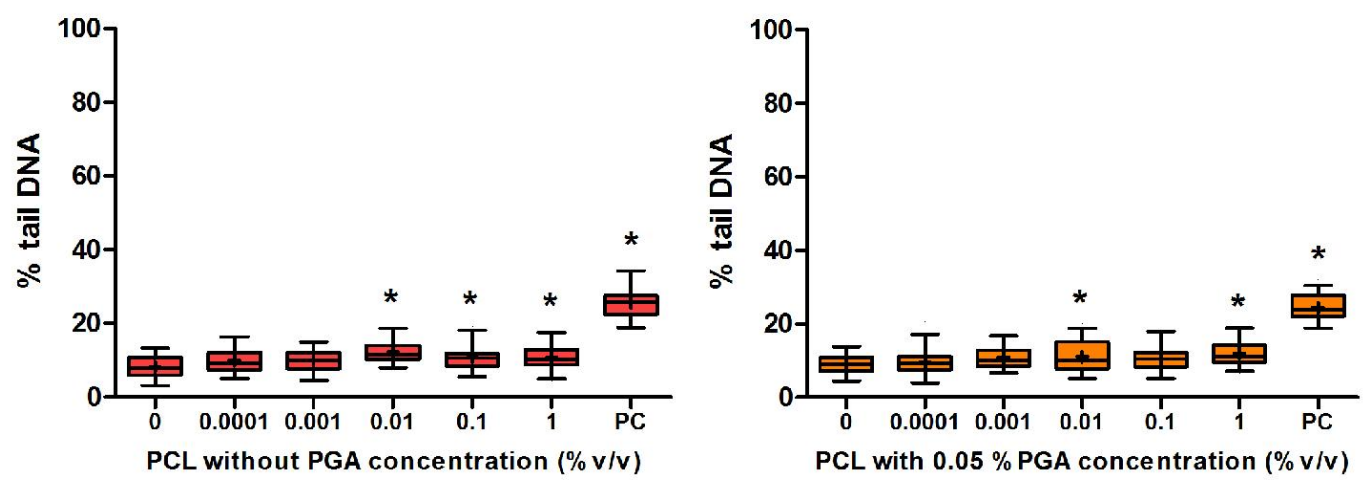

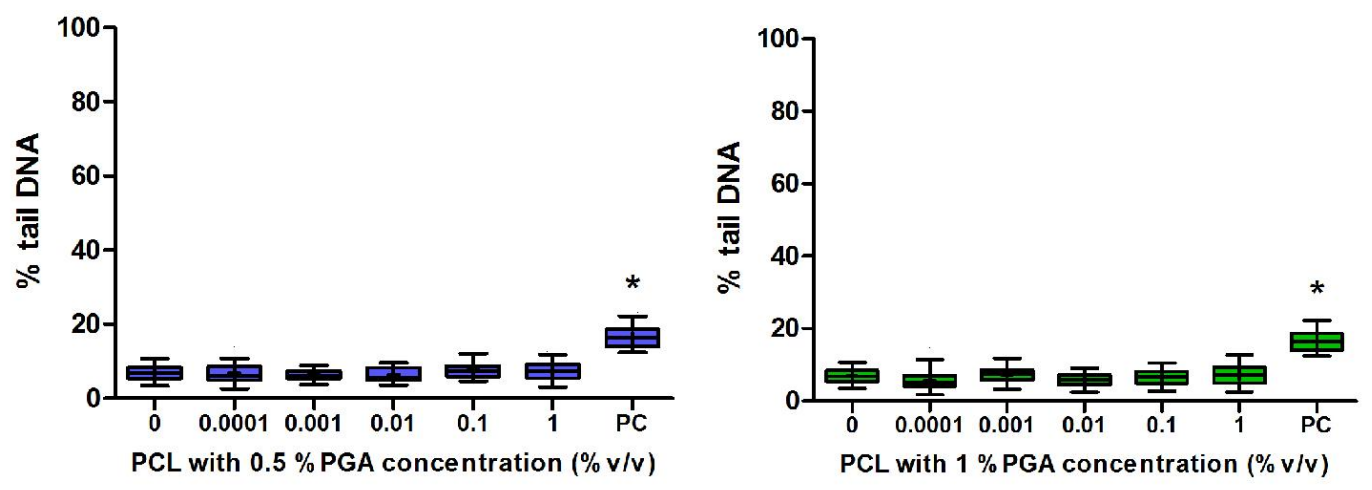

Figure 8. DNA strand break induction in HepG2 cells by samples: PCL without PGA, PCL with $0.05 \%$ PGA, PCL with $0.5 \%$ PGA and PCL with 1\% PGA. The DNA damage was assessed using the comet assay as described in Materials and methods, and expressed as \% of DNA in the tail. Fifty cells were analyzed per experimental point in each of the three independent experiments. Data are presented as quantile box plots. The edges of the box represent the $25^{\text {th }}$ and $75^{\text {th }}$ percentiles, the median is a solid line through the box, mean values are represented as square $(+)$, and the error bars represent the $95 \%$ confidence intervals. $* \mathbf{P}<\mathbf{0 . 0 5}$ and $* * * \mathbf{P}<\mathbf{0 . 0 0 1}$ denotes a significant difference between PCL NPstreated and control groups using ANOVA, Kruskal-Wallis with Dunnet's post test.

The exposure of HepG2 cells to sample of PCL without PGA induced slight, though statistically significant increase in DNA strand breaks at concentrations $0.01,0.1$ and $1 \%$ and the exposure to a sample of PCL with $0.05 \%$ PGA induced an increase in DNA strand breaks at concentrations 0.01 and $1 \%$. On the other hand, samples PCL with $0.5 \%$ PGA and $1 \%$ PGA did not cause statistically significant DNA damage (Figure 8). These data show that the samples of PCL without PGA lead to higher levels of DNA strand breaks than the samples with PGA. It is possible that PGA has a protective effect against genotoxicity, however further studies are needed to firmly prove this hypothesis [54]. While analyzing literature data, we have found only one study on the genotoxicity of blank PCL nanoparticles [55] which demonstrates that PCL nanoparticles are not genotoxic in rats in vivo after three months, as well as twelve months of intraperitoneal implantation. In that study, 8-hydroxy-2'deoxy guanosine and chromosomal aberrations were used as genotoxicity markers. However, the study does not provide the concentration of PCL nanoparticles and the characteristics of PCL nanoparticles. In other studies using PCL nanocapsules as carrier systems for herbicides [56] and nanoparticles as a combination of PCL-PEG-PCL [57], authors, who used a battery of assays, demonstrate that PCL is not genotoxic in these combinations.

\section{CONCLUSION}

In this study, we describe a new method for obtaining smooth and uniform PCL nanospheres with sizes below $200 \mathrm{~nm}$. The method itself has a few advantages concerning freeze drying. Due to the right choice of the stabilizing agent - PGA, we have managed to avoid the use of additives, as well as to shorten the duration of the process. We have also determined that PGA concentrations above $0.05 \%$ have negative effects on the morphology in terms of affecting the particles size, agglomeration, irregular shapes and their nonuniformity. Therefore, the best concentration of PGA for the mentioned system is $0.05 \%(\mathrm{w} / \mathrm{w})$. All samples prepared with PGA show no cytotoxicity, a low potential for ROS generation and a low genotoxicity potential; all of 
these factors are crucial for good biocompatibility. We have also found that no spherical particles were formed when a one-percent saccharose solution is used as an excipient. Instead, PCL micron-size plates and cubes of various sizes are formed.

\section{Acknowledgement}

This study was supported by the Ministry of Education, Science and Technological Development of the Republic of Serbia, under Grant No. III45004: Molecular designing of nanoparticles with controlled morphological and physicochemical characteristics and functional materials based on them. The authors would like to thank to Aleksandra Radulović and Branimir Kovačević for FTIR measurements and Miloš Bokorov for SEM measurements. 


\section{References}

[1] S.K. Soppimath, T.M. Aminabhavi, A.R. Kulkarni and W. E. Rudzinski, J. Control. Release., 70 (2001) 120.

[2] U. Edlund, in A.-C. Albertsson (Ed.), Degradable Aliphatic Polyesters, Vol. 157, Springer, Berlin, 2002, Chapter 3.

[3] O. Coulembier, P. Degée, J.L. Hedrick and Philippe Dubois, Prog. Polym. Sci., 31 (2006) 723-747.

[4] A. Kumari, S. K. Yadav and S.C. Yadav, Colloids Surf. B Biointerfaces., 75 (2010) 1-18.

[5] V.R. Sinha, K. Bansal, R. Kaushik, R. Kumria and A. Trehan, Int. J. Pharm., 278 (2004) 1-23.

[6] M.A. Woodruff and D.W. Hutmacher, Prog. Polym. Sci., 35 (2010) 1217-1256.

[7] J.P. Rao and K.E. Geckeler, Prog. Polym. Sci., 36 (2011) 887-913.

[8] T.K. Dash and V.B. Konkimalla, J. Control. Release., 158 (2011) 15-33.

[9] M.R. Abertura, S. J. Molpeceres, M. Guzmaán and F. Garciáa, J. Microencapsul., 19 (2002) 61-72.

[10] J.L. Arias, M.Lopez-Viota, E. Sáez-Fernández and M.A. Ruiz, Colloids Surf. B Biointerfaces., 75 (2006) 204-208.

[11] L.H. Guerreiro, D.D. Silva, E. Ricci-Junior, W. Girard-Dias, C.M. Mascarenhas, Mauro Sola-Penna, K. Miranda and L.M. Lima, Colloids Surf. B Biointerfaces., 94 (2012) 101.

[12] M.K. Lee, M.Y. Kim, S. Kim, J. Lee, J. Pharm. Sci., 98 (2009) 4808-4817.

[13] A. Saez, M. Guzmaán, J. Molpeceres and M.R. Aberturas, Eur. J. Pharm. Biopharm., 50 (2000) 379-387.

[14] W. Abdelwahed, G. Degobert, S. Stainmesse and H. Fessi, Adv. Drug Deliv. Rev., 58 (2006) 16881713.

[15] W. Abdelwahed, G. Degobert and H. Fessi, Int. J. Pharm., 309 (2006) 178-188.

[16] W. Abdelwahed, G. Degobert and H. Fessi, Eur. J. Pharm. Biopharm., 63 (2006) 87-94.

[17] S.R. Schaffazick, A.R.Pohlmann, T. Dalla-Costa and S.S. Guterres, Eur. J. Pharm. Biopharm., 56 (2003) 501-505.

[18] W. Abdelwahed, G. Degobert and H. Fessi, Int. J. Pharm., 324 (2006) 74-82.

[19] A. Gajewicz, B. Rasulev, T.C. Dinadayalane, P. Urbaszek, T. Puzyn, D. Leszczynska and J.Leszczynski, Adv. Drug Deliv. Rev., 64 (2012) 1663-1693.

[20] T. Tamiya, N. Okahashi, R. Sakuma, T. Aoyama, T. Akahane and J.J. Matsumoto, Cryobiology, 22 (1985) 446-456.

[21] K. Seguro, T. Tamiya, T. Tsuchiya and J.J. Matsumoto, Cryobiology, 27 (1990) 70-79.

[22] M. Mitsuiki, A.Mizuno, H. Tanimoto, and M. Motoki, J. Agric. Food Chem., 46 (1998) 891-895.

[23] J.M. Buescher and A. Margaritis, Crit. Rev. Biotechnol., 27 (2007) 1-19.

[24] I-L. Shih and Y-T. Van, Bioresour. Technol., 79 (2001) 207-225.

[25] C. Li, X. Wen, E.F. Jackson and W. Sidney, Poly(L-glutamic acid) paramagnetic material complex and use as biodegradableMRI contrast agent, Patent Cooperation Treaty Application Number WO2005063304, 2005.

[26] M. Stevanović, B. Kovačević, J. Petković, M. Filipič and D. Uskoković, Int. J. Nanomedicine, 6 (2011) 2837-2847. 
[27] M. Stevanović, V. Uskoković, M. Filipović, S. D.Škapin, D. Uskoković, ACS Appl. Mater. Interfaces., 5 (18) (2013) 9034-9042.

[28] A. S. Chan, C. H. Chen, C. M. Huang, M. F. Hsieh, J. Nanosci. Nanotechnol., 10(10) (2010) 6283-6297.

[29] N. Filipović, M. Stevanović, A. Radulović, V. Pavlović, and D. Uskoković, Compos. Part B: Eng., 45 (2013) 1471-1479.

[30] T. Mossman, J. Immunol. Methods, 65 (1983) 55-63.

[31] M. Stevanović,T. Maksin,J. Petković,M. Filipič and D. Uskoković, Nanotechnology, 20 (2009) $335102 / 1-12$.

[32] R.A. Osseni,C. Debbasch,M.O. Christen, P. Rat and J.M. Warnet, Toxicol. In Vitro., 13 (1999) 683-688.

[33] J. Petković, B. Žegura, M. Stevanović, N. Drnovšek, D. Uskoković,S. Novak and M. Filipič, Nanotoxicology, 5 (2011) 341-353.

[34] N.P. Singh, M.T. McCoy, R.R. Tice and E.L. Schneider, Exp. Cell. Res., 175 (1988) 184-191.

[35] G.H. Ho, T.I. Ho, K.H. Hsieh, Y.C. Su, P.Y. Lin, J. Yang, K.H. Yang and S.C. Yang, J. Chin. Chem. Soc., 53 (2006) 1363-1384.

[36] T. Elzein,. M. Nasser-Eddine, C. Delaite, S. Bistac, and P. Dumas, J. Colloid. Interface Sci., 273 (2004) 381-387.

[37] S. Suganya, T. Senthil Ram, B. S. Lakshmi and V. R. Giridev, J. Appl. Polym. Sci., 121(2011) 28932899.

[38] K. Arai and R. Takashi, Bull. Jpn. Soc. Sci. Fish., 39 (1973) 533- 541.

[39] H. Fessi, F. Puisieux, J.P. Devissaguet, N. Ammoury, S. Benita, Int. J. Pharm., 5 (1989) R1-R4.

[40] W. Wang, Int. J. Pharm., 203 (2000) 1-60.

[41] M. J. Pikal, 1999. Mechanisms of protein stabilization during freeze-drying and storage: the relative importance of thermodynamic stabilization and glassy state relaxation dynamics. In: Rey, L., May, J.C. (Eds.), Freeze-Drying:Lyophilization of Pharmaceutical and Biological Products, vol. 96. Marcel Dekker, New York, pp. 161-198.

[42] R. Liu, L. J. Am. Chem. Soc., 119 (20) (1997) 4791-4792.

[43] J. Liu, T. Viverette, M. Virgin, M. Anderson and P. Dalal, Pharm. Dev. Technol., 10 (2005) 261-272.

[44] N.-O. Chung, M.K. Lee and J. Lee, Int. J. Pharm., 437 (2012) 42- 50.

[45] O.Y. Kurapova,V.G. Konakov, S.N. Golubev, V.M. Ushakov and I.Y. Archakov, Rev. Adv. Mater. Sci., 32 (2012) 112-132.

[46] Y. Roos, M. Karel, Phase transitions of amorphous sucrose and frozen sucrose solutions, J. Food Sci., 56 (1991) 266-267.

[47] J. Shi, Steric Stabilization, CISM, Columbus, U.S. 2002.

[48] U. Kedar, P. Phutane, S. Shidhaye, and V. Kadam, Nanomedicine, 6 (2010) 714-729.

[49] R. P. Singh, P. Ramarao, Toxicol. Sci., 136(1) (2013)131-143.

[50] D. Kirkland, S. Pfuhler, D. Tweats, M. Aardema, R. Corvi, F. Darroudi, A. Elhajouji, H. Glatt, P. Hastwell, M. Hayashi, P. Kasper, S. Kirchner, A. Lynch, D. Marzin, D. Maurici, J. R. Meunier, L. Muller, G. Nohynek, J. Parry, E. Parry, V. Thybaud, R. Tice, J. van Benthem, P. Vanparys, and P. White, Mutat Res., 628(1) (2007) 31-55. 
[51] S. Knasmuller, W. Parzefall, R. Sanyal, S. Ecker, C. Schwab, M. Uhl, V. Mersch-Sundermann, G. Williamson, G. Hietsch, T. Langer, F. Darroudi, and A. T. Natarajan, Mutat Res., 402(1-2) (1998) 185-202.

[52] E. V. R. Campos, N. F. Silva de Melo, V. A. Guilherme, E. de Paula, A. H. Rosa, D. R. de Araújo, L. F. Fraceto, J Pharm Sci., 102(1) (2013) 215-226.

[53] S. Venitt, and D. H. Philips, The Importance of Environmental Mutagens in Human Carcinogenesis and Germline Mutations. In Environmental Mutagenesis. Eds. D. H. Philips and S. Venitt., Bios Scientific Press. Oxford, (1995).

[54] Chen J, Herrup K, PloS one (2012) 7(3):e33177 doi:10.1371/journal.pone.0033177

[55] D. Surendran, R. S. Sarath Kumar, C. S. Geetha, P. V. Mohanan, BIO., 2 (2012) 37-46.

[56] R. Grillo, N. Z. dos Santos, C. R. Maruyama, A. H. Rosa, R. de Lima, L. F. Fraceto, J Hazard Mater., 231-232 (2012) 1-9.

[57] Y. Huang, H. Gao, M. Gou, H. Ye, Y. Liu, Y. Gao, F. Peng, Z. Qian, X. Cen, Y. Zhao, Mutat Res., 696(2) (2010) 101-106. 